\title{
'A NEW GENERATION OF DENTAL NANOMATERIALS COULD SOON OFFER ENHANCED STRENGTH AND CARIES PROTECTION'
}

\begin{abstract}
Dr Michele Barbour is Senior Lecturer in Dental Biomaterials at the University of Bristol. Michele's research group is focussed on oral nanoscience, exploiting developments in nanotechnology to confer improved antimicrobial and mechanical properties on dental and other biomaterials. She is also deputy head of Bristol Dental School.
\end{abstract}

\section{You studied physics at university, what led you to specialise in oral nanoscience?}

The part of my undergraduate degree I enjoyed the most was the research project in the final year. To have the opportunity to do real, meaningful science, to obtain and analyse data that could help to further the understanding of the field, and all while still an undergraduate: it felt like such a privilege. I spent time in atmospheric and planetary physics, working on a project to design a remote sensing device to be sent to orbit Mars, and in atomic and laser physics, developing a laser device to study processes occurring inside combustion engines in real time. I came to the end of this period with two strong convictions: 1) that I wanted to pursue a career in research, and 2) that I wanted to move away from physics as a discipline and instead use my physical sciences training to address a question related to human health. I looked at a lot of different projects, and ultimately chose to come to Bristol dental school to work in what was then the dental materials group. I found myself rubbing shoulders at the lab bench with chemists, materials scientists and dentists as well as physicists. It was an energising experience. This time gave me a taste for working in an interdisciplinary research environment. That's pretty standard now - the days of physicists doing physics with other physicists in a
'Clinicians should be engaged with the research underpinning the materials available to them...' big building labelled 'Physics' are largely behind us - but in the early 2000s it was still comparatively unusual. Around the time of my PhD, nanoscience and nanotechnology were booming. It became apparent that nanoscience had huge potential in many fields, and I wanted to take those new technologies and concepts and apply them to dentistry. So when I got my first lectureship and founded my research group, oral nanoscience most accurately described what I wanted to achieve.

\section{What surprised you most about working in a dental school?}

The hierarchical structure; the deference afforded to people at a 'higher' level than oneself. As an undergraduate in the physics department at Oxford, everyone was on first name terms. I distinctly remember calling the head of department - a very eminent laser physicist - Colin. There was none of this 'Dr This' and 'Professor That' business. I think maybe I shocked a couple of people at first, by making the assumption that it was fine to address senior staff by their first names! I adjusted my approach to fit with the culture at the time but now I have my own students, I don't perpetuate the tradition. My undergraduates call me Michele and that's the way I prefer it - it's possible to have authority and gravitas but still be approachable and human.

\section{What are you working on at the moment?}

My group and I are working on a suite of novel antimicrobial nanoparticles. These have been in
Michele will be giving this year's BDA-BDJ Winter Lecture in London on the 10th December 2014. Tickets are complimentary for BDA members and for this popular event tickets will be allocated by a ballot which closes on 19 November. To apply, visit www.bda.org/winterlecture. Michele's Winter Lecture will be on the topic of Dental materials today and tomorrow. One hour of verifiable continuing professional development (CPD) is available for those attending and a recording of the lecture will be made available after the event for all members to view online. The event is sponsored by the Wrigley Oral Healthcare Programme and a festive reception will follow the lecture.

development for a few years, but the university was understandably keen to protect the intellectual property, so we had to file a patent application before we could publicise our work widely. Now the publishing embargo is lifted we can shout about it from the rooftops!

Our nanoparticles act as a slow release device for chlorhexidine. As biocides go, chlorhexidine is pretty cheap, readily available, and relatively straightforward from a regulatory point of view as it's already so widely used. The difference between our nanoparticle technology and the established ways of using chlorhexidine is that we can sustain a steady release of chlorhexidine for several weeks or months, creating a locally antimicrobial environment for an extended period, rather than the few hours that can be achieved using conventional approaches.

We're using the chlorhexidine nanoparticles as coatings and dopants for a wide range of materials. For instance, the Medical Research Council is supporting a project where we incorporate the nanoparticles into a glass-ionomer cement, the aim being to create a restorative material that can leach very low concentrations of chlorhexidine over an extended period, yielding a sustained antimicrobial environment without imparting an unpleasant taste or propensity for staining. That project's going really well - we've achieved chlorhexidine release for 6 months and that's without any 
topical application of chlorhexidine rinse, and without compromising the strength of the material. Concurrent with this we're investigating recharge capacity, and hope to be able to develop a material where the patient could, say, use a chlorhexidine mouthrinse once or twice a year to 'top up' the chlorhexidine nanoparticle reservoir in the cement.

\section{What are you most proud of so far in your career?}

Students. I have to stop myself saying 'my' students! I love to work with students - it's hard not to feel proprietorial towards them at times. I have supervised 21 postgraduate research students to date. The relationship between supervisor and research student is a special one - you act as advisor, mentor, champion, challenger, administrator, career coach, examiner and protector. A PhD student has to find their feet, develop their independence, forge their way forward in science and in life, but they also sometimes need to be guided around pitfalls and away from rabbit holes, and encouraged to stand up to tasks or people they find daunting. It's a delicate balancing act and of course every student has their own needs, their own challenges to face. But it's a huge pleasure and privilege to work with bright and ambitious young people, and to observe and support their development during a formative period in their lives. I also derive great pleasure from working with undergraduate students. I teach dental materials to BDS students in years 2-4. I also provide a suite of dental materials e-learning podcasts for on-demand use throughout the BDS. I have been told by 5th year students that they have these 'on continuous loop in the kitchen' in the run-up to finals! I'm tickled to picture my disembodied voice explaining the finer points of the setting reactions of glass ionomers while the students are drinking tea or cooking pasta.

In all seriousness, BDS students are exceptionally well-motivated and focused. They need to be - there's a lot of competition to get into dental school, and I'd like to think that the feeling of achievement of having gained entry can help to carry students through the rigours of what is undoubtedly a tough degree. They're a diverse bunch, both in terms of geographical and socioeconomic background, but they are united by a shared ambition to improve their patients lives. To support them while they metamorphosise from academically accomplished, aspirational but inexperienced and sometimes naive individuals, into professionals with a wealth of skills, experience, confidence and maturity - it never fails to fill me with pride and joy.

\section{How important do you think it is for clinicians to be engaged with scientific research?}

It's a duty; it's fundamental. Inevitably I look at this from a biomaterials perspective, but the same can be said of medicines, understanding of disease processes, and the impact of lifestyle factors on oral health. I tell my second year undergraduates that many of the materials they will see on the shelves today will not be available when they graduate, never mind in 10, 20, 30 years' time. Materials develop, sometimes for the better and sometimes less so, but it's essential that the clinician keeps up to date with the scientific concepts that underpin the materials available to them, and the evidence base informing their use. When I'm asked a question like this, I sometimes find it useful to turn the question around, to ask the opposite. So in this case: 'Does it matter if a clinician doesn't engage with research?' That's pretty straightforward. Would I want to visit a dentist who is aware of and understands modern materials, and how they are best selected and applied? Would I be happy to see a dentist who uses only materials that date from the year they graduated? Or one who uses modern materials but without having any insight into their properties and function? I know which I would choose!

\section{Do you feel that research in the field of oral nanoscience is being successfully translated to practice?}

I don't think this kind of translational research is as well funded as it merits. I see some improvements to the funding environment, but there's an extent to which you have to consider how the funding bodies prioritise applications. If you seek funding for a project to develop an improved restorative material which will potentially benefit the oral health of millions of patients, and in competition with your grant proposal is a project to develop an improved heart valve which will benefit a much smaller group of patients but to a more profound degree, then it's perhaps not surprising that some funders will plump for the savea-few-lives over the improve-many-lives option. One has to understand that and, arguably, embrace it. Ultimately, much translational research in dentistry requires the financial power of a manufacturer. One has to proceed carefully but I have had some great experiences of working in mutually beneficial, science-driven and patient-focused partnerships with companies. Nowadays there is a much better appreciation in the university research community that to take technologies from bench to bedside it is essential to work in partnership with industry.

\section{What do you think is the biggest challenge facing biomaterials research?}

Specific to dental biomaterials, the big challenge right now is the phase down of amalgam following the Minamata Convention, and the development of materials that we can use in its place. In a broader sense,
I think that the biggest challenge facing biomaterials research is microbial resistance to antibiotics. The ramifications of the imminent failure of many of our front-line antibiotics are huge. New antibiotics are costly and difficult to develop and in one sense may simply be pushing the problem on a few years rather than solving it, as microbes become resistant to new antibiotics. We need a different approach, and I think antimicrobial biomaterials, which prevent infection at the site of surgery or implantation, are a good candidate for this.

\section{What is the future for nanoscience and nanotechnology in dentistry?}

Nanotechnology has been in use in dentistry for decades. Silica fillers for composites with a diameter of $50 \mathrm{~nm}$ were developed in 1978 - but this was before the widespread use of the 'nano' prefix and they were called 'ultrafine' fillers. It was a few years before the marketing folks thought to write 'nano!' on the box, but nanofilled composites have been on the market and in patients' mouths for decades. So in one sense, the use of nanotechnology in dental materials is not new. What is new is the understanding of some of the benefits that nanoscale technology can offer; the additional function that can be wrought by embracing developments in the manipulation and understanding of matter at this scale, and the scale-up of the processes that make it a viable proposition for bulk production.

But I'm dodging the question! What's really the future? I'd like to think that we will soon see materials that support the phase down of amalgam by offering bulk cure, strong, durable restorations with acceptable aesthetics and cost. Nanoscience has a lot to offer this challenge, for instance the use of structurally reinforcing nanofibres and nanowhiskers. I'd also like to see restorative materials that offer a genuine, sustained protection against secondary caries on the market in the next five years, and this could occur by the use of antimicrobial nanoparticles. This is achievable from a scientific point of view but will need engagement from key stakeholders to make it happen. 